ISASE 2020

\title{
Emotions of Simplified and Traditional Chinese Typeface
}

\author{
Qianru QIU *, Shu WATANABE ** and Kengo OMURA *** \\ * Fuji Xerox Co. Ltd, 6-1 Minatomirai, Nishi-ku, Yokohama-shi, Kanagawa-ken, 220-8668, Japan \\ seijo.qiu@fujixerox.co.jp \\ ** Fuji Xerox Co. Ltd, 6-1 Minatomirai, Nishi-ku, Yokohama-shi, Kanagawa-ken, 220-8668, Japan \\ shu.watanabe@fujixerox.co.jp \\ *** Fuji Xerox Co. Ltd, 6-1 Minatomirai, Nishi-ku, Yokohama-shi, Kanagawa-ken, 220-8668, Japan \\ kengo.omura@fujixerox.co.jp
}

\begin{abstract}
Chinese typeface is widely used in design works that have numbers of users. The majority of Chinese words today consist of two or more characters. Basically, the simplified forms of Chinese characters are used in mainland China, Singapore, and Malaysia. The corresponding traditional characters are used in Taiwan, Hong Kong, and Macau. This study quantifies the relationships between emotions and Chinese typefaces by the process of kansei engineering. 20 simplified types and 20 traditional types were extracted and the kansei evaluation experiment was conducted for the people in mainland China and Taiwan where are the main regions using Chinese. The results of cluster analysis, factor analysis, and dual scaling analysis are used for comparison of affective differences between mainland China and Taiwan. Particularly, it is found that for the widely used types Heiti, Songti, and Kaiti, the people in mainland China and Taiwan have opposite feelings of classic and contemporary.
\end{abstract}

Keywords: Font emotion, Affective design, Chinese Characters

\section{INTRODUCTION}

Typeface is fundamental to document designs. As a visual element of the document, type affects the reader's initial impression and reading experience [1]. It is proved that typefaces have significant impacts on human emotions in the research of document design [2]. Nowadays, the needs of multilingual design are growing for multilingual signage, website, etc. There are some researches about affective design of Latin type, Japanese type, Chinese type, etc. $[3,4,5,6]$. The affective knowledge of typeface is helpful to font selection in design works, even for document designers and graphic artists.

Chinese characters are among the most widely adopted writing systems in the world by numbers of users. It is used not only in mainland China, Taiwan, and Hong Kong, but Japan, North Korea, South Korea, and Vietnam, although Mongolia and parts of Central Asia. The standard of Chinese characters in these areas are settled independently. The so-called Chinese typeface contains simplified Chinese (SC) used in mainland China and traditional Chinese (TC) used in Taiwan. As we know, there are differences in the perception of typeface personalities between varying cultures, gender, and expertise-level. To our knowledge, there are less research about the affective differences between SC and TC types. In other words, there are less knowledge about the typographic affective differences between the people in mainland China and Taiwan.

This research aims to evaluate the affective performance of typical Chinese typefaces by quantitative methods and compare the different impressions for typefaces between the people living in mainland China and Taiwan. We extract some typical typefaces of SC and $\mathrm{TC}$ fonts, and conduct the kansei evaluation experiment to gain the affective evaluation data for these fonts. In this work, the evaluation results are shown as a guideline for affective font selection. Moreover, cluster analysis, factor analysis and dual scaling analysis are executed for comparison.

\section{AFFECTIVE EVALUATION FOR TYPEFACES}

\subsection{Font selection}

For SC and TC types, the Chinese characters share the same glyphs and some have subtle differences. Even some punctuations have some differences in design, while it is not strict in daily use. Although there are thousands of Chinese fonts, they can be basically classified into several groups, as Heiti, Yuanti, Songti, Fangsong, Kaiti, other calligraphy types and Decorative types in digital age. In 
Table 1: Font list of SC and TC (some fonts have SC and TC in a file, some have different font file for SC and TC)

\begin{tabular}{|c|c|c|c|c|}
\hline classification & SC fonts & Sample & TC fonts & Sample \\
\hline \multirow{6}{*}{ Heiti } & Heiti & 论语 & Heiti & 論語 \\
\hline & $\overline{\text { SimHei }}$ & 论语 & SimHei & 論語 \\
\hline & \multicolumn{2}{|c|}{ Hiragino Sans GB 论语 } & \multicolumn{2}{|c|}{ Hiragino Sans GB 論語 } \\
\hline & MS Yahei & 论语 & MS Yahei & 論語 \\
\hline & LantingHei & 论语 & LantingHei & 論語 \\
\hline & Source Han Sans & 论语 & Source Han Sans & 論語 \\
\hline \multirow{2}{*}{ Yuanti } & Yuanti & 论语 & Yuanti & 論語 \\
\hline & DFYuanti & 论语 & DFPGYuanti & 論語 \\
\hline \multirow{2}{*}{ Songti } & Songti & 论语 & Songti & 論語 \\
\hline & SimSun & 论语 & SimSun & 論語 \\
\hline \multirow{2}{*}{ Fangsong } & STFangsong & 论语 & STFangsong & 論語 \\
\hline & Adobe Fangsong & 论语 & Adobe Fangsong & 論語 \\
\hline Kaiti & Kaiti & 论语 & Kaiti & 論語 \\
\hline Seal script & DFPJinWne & 论语 & DFPGJinWen & 論語 \\
\hline $\begin{array}{l}\text { Semi-cursive } \\
\text { script }\end{array}$ & - & & DFPGXingshu & 論語 \\
\hline Weikai & DFPTanLi & 论语 & - & \\
\hline Clerical script & Weibei & 论语 & Weibei & 論語 \\
\hline \multirow{4}{*}{ Decorative type } & DFPPOP & 论语 & DFPGPOP & 論語 \\
\hline & DFPShaoNv & 论语 & DFPGGirl & 論語 \\
\hline & Wawati & i论语 & Wawati & 論語 \\
\hline & HanziPen & 论语 & HanziPen & 論語 \\
\hline
\end{tabular}

this work, $20 \mathrm{SC}$ fonts and $20 \mathrm{TC}$ fonts are selected shown as Table 1. Among them, 19 fonts are belonging with the same types, and 1 font is different. These fonts are from some famous Chinese font company, as Sino Type (ST), Dyna Font (DF), etc. Among them, 6 fonts of Heiti, 2 fonts for Yuanti, Songti, and Fangsong which are commonly used in digital devices are selected.

\subsection{Kansei evaluation experiment}

For the evaluation targets, one chapter of the Analects of Confucius is selected as the content of samples which is widely known in China. Leading is set to $1.6 \mathrm{em}$. $20 \mathrm{SC}$ samples and $20 \mathrm{TC}$ samples were made for each SC and TC fonts shown as Figure 1. 30 kansei words were extracted to express design tastes of typography, as pretty (漂亮), casual (随性), dynamic (动感), romantic (浪漫), mild (温和), feminine (女人味), natural (自然), elegant (优雅), gorgeous (华丽), wild (豪放), classic (古典), formal (正式), dandy (花俏), chic (别致), fresh (清新), clear (清晰), modern (时髦), pop (流行), retro (怀旧), noble (高贵), friendly (亲切), contemporary (现代), standard (标准), stylish (时尚), expressive (生动), readable (易读), like (喜欢), reliable (可靠), attractive (醒目), beautiful (美丽). All these kansei words are used for evaluation experiment of SC and TC typefaces. The experiments for $\mathrm{SC}$ and $\mathrm{TC}$ were conducted separately.

\begin{tabular}{|l|}
\hline 学而时习之, 不亦说乎? \\
有朋自远方来, 不亦乐乎? \\
人不知而不愠, 不亦君子乎? \\
\hline 學而時習之, 不亦說乎? \\
有朋自遠方來, 不亦樂乎? \\
人不知而不慍, 不亦君子乎? \\
\hline
\end{tabular}

Figure 1: Evaluation samples of SC and TC in Adobe Fangsong

The investigation was executed by 5-point scales of SD method and entrusted to the investigation company, Macromill. It was conducted for 82 people in mainland China to evaluate SC samples and 82 people in Taiwan to evaluate TC samples. The age of these participants had a distribution in twenties, thirties, forties, and fifties. The evaluation results were collected in January, 2017.

\section{ANALYSIS AND RESULTS}

\subsection{Cluster analysis}

Ward method is used for hierarchical cluster analysis. As the results, $20 \mathrm{SC}$ fonts can be divided into 3 groups which are close to serif, san-serif, and design type in Latin types. Here, it is intuitive to use serif and san-serif for naming font classifications and the design type class includes Calligraphy and decorative types. The details are as follows, where ' $[$ ]' denotes sub-classifications that have close images.

- Serif: [Adobe Fangsong, SimSun, STFangsong, Kaiti], [DF Yuanti, Songti]

- San-serif: [Heiti, LantingHei, SimHei, Source Han Sans, Yuanti], [Hiragino Sans, MS YaHei]

- Design type: [DFPPOP], [Weibei, DFPTanLi], [DFPJinWen], [HanziPen, DFPShaoNv], [Wawati]

$20 \mathrm{TC}$ fonts have different classifications from SC fonts that Fangsong and Kaiti are evaluated with closer tastes of calligraphy, and Heiti, Songti, Yuanti have close tastes which are widely used in modern digital world. They are divided into 3 groups denoted as calligraphy type, design type, and practical modern type. The details are as follows, where '[]' denotes sub-classifications that have close images.

- Calligraphy type: [Adobe Fangsong, Kaiti, Weibei], [DFPGXingshu] 
- Design type: [DFPGJinWen], [DFPGPOP], [HanziPen], [WAwati, DFPGGirl]

- Practical modern type: [DFPGYuanti, Yuanti], [Heiti, MS YaHei, SimHei, Hiragino Sans, Source Han Sans, LantingHei], [SimSun, Songti, STFangsong]

\subsection{Factor analysis}

In this evaluation experiment, 19 fonts for $\mathrm{SC}$ and $\mathrm{TC}$ types are same among 20 evaluated fonts. To get the main kansei factors of Chinese typeface, the affective evaluation data of these 19 fonts from the subjects of mainland China and Taiwan is integrated. The results of factor analysis by promax rotation method are shown in Table 2. Factor 1 is interpreted as Informality with opposing meaning of casual and formal. Factor 2 is interpreted as Practicality denoting the word is readable or not. Factor 3 is interpreted as Times that classic, retro, and contemporary imply epochal meanings.

Table 2: Factor loadings by promax rotation

\begin{tabular}{|c|c|c|c|c|}
\hline \multicolumn{2}{|c|}{ Kansei words } & Factor1 & Factor2 & Factor3 \\
\hline \multirow{12}{*}{ 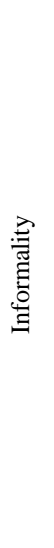 } & casual & 1.0707 & -0.1672 & -0.2233 \\
\hline & dynamic & 1.0547 & -0.2776 & -0.0280 \\
\hline & dandy & 1.0182 & -0.4133 & -0.0665 \\
\hline & modern & 0.9836 & 0.0225 & -0.0189 \\
\hline & expressive & 0.9760 & -0.0895 & 0.0355 \\
\hline & pop & 0.8727 & 0.2954 & -0.1090 \\
\hline & romantic & 0.8398 & -0.0071 & 0.2140 \\
\hline & stylish & 0.8148 & 0.2577 & 0.0622 \\
\hline & wild & 0.7629 & 0.1832 & 0.1205 \\
\hline & chic & 0.7532 & -0.1487 & 0.3511 \\
\hline & feminine & 0.7527 & 0.2374 & 0.1441 \\
\hline & beautiful & 0.4800 & 0.2711 & 0.4552 \\
\hline \multirow{13}{*}{ 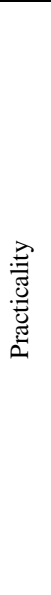 } & readable & -0.1929 & 1.1010 & -0.1524 \\
\hline & standard & -0.4485 & 1.0469 & 0.0315 \\
\hline & contemporary & 0.3434 & 0.9763 & -0.3378 \\
\hline & clear & -0.0219 & 0.9585 & 0.0265 \\
\hline & formal & -0.4324 & 0.9267 & 0.2034 \\
\hline & reliable & -0.1193 & 0.8676 & 0.2449 \\
\hline & attractive & 0.1648 & 0.7403 & 0.0792 \\
\hline & natural & 0.4431 & 0.6546 & 0.0757 \\
\hline & friendly & 0.5945 & 0.6407 & -0.1227 \\
\hline & like & 0.3901 & 0.6390 & 0.1676 \\
\hline & mild & 0.5345 & 0.5818 & -0.0141 \\
\hline & fresh & 0.4685 & 0.5078 & 0.2123 \\
\hline & pretty & 0.3568 & 0.4163 & 0.4011 \\
\hline \multirow{5}{*}{$\stackrel{\mathscr{\Xi}}{\Xi}$} & classic & -0.1566 & -0.1587 & 1.1043 \\
\hline & retro & -0.0186 & -0.0235 & 0.9800 \\
\hline & noble & 0.1041 & 0.2690 & 0.7511 \\
\hline & elegant & 0.2740 & 0.2580 & 0.6079 \\
\hline & gorgeous & 0.5228 & 0.0895 & 0.5634 \\
\hline
\end{tabular}

In addition, from the correlation coefficients among these kansei words we found that the people in mainland China and Taiwan have relatively close impressions of standard, formal, reliable, readable, clear, dandy, casual, contemporary, attractive, dynamic, elegant, romantic, and mild. Most of these words are objective feelings to fonts. On the other hand, some more subjective feelings which are effected by culture, life environment and experience as classic, pretty, natural, chic, fresh, like, noble, retro, friendly, gorgeous, beautiful, expressive, modern, stylish, feminine, wild, and pop, are not significant correlated between the people in mainland China and Taiwan.

\subsection{Comparison of mainland China and Taiwan}

To map all fonts and emotions, dual scaling analysis are executed. Optimal weights were calculated using the cross table containing the evaluation value of each kansei word to each font. After weighting to reflect the relative

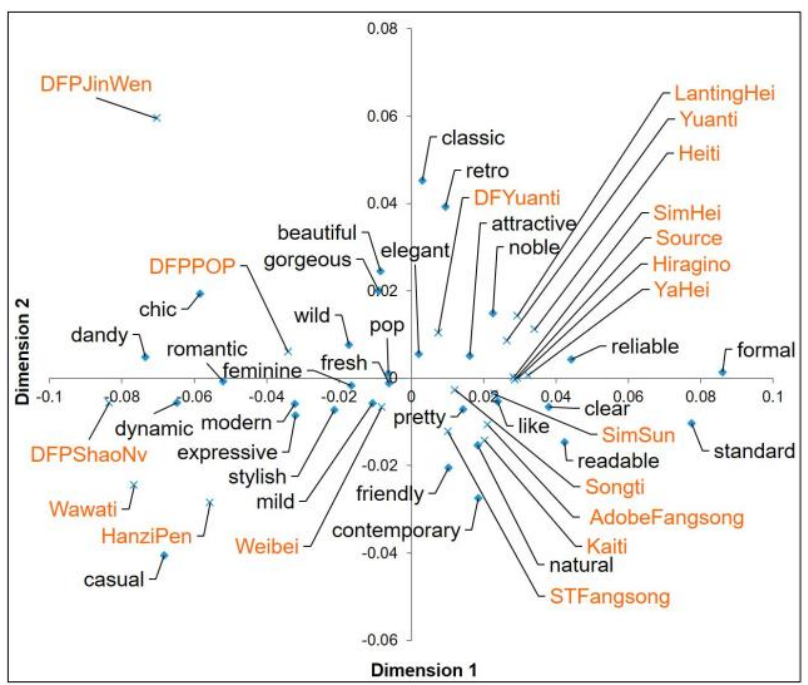

Figure 2: Emotional mapping of $20 \mathrm{SC}$ fonts

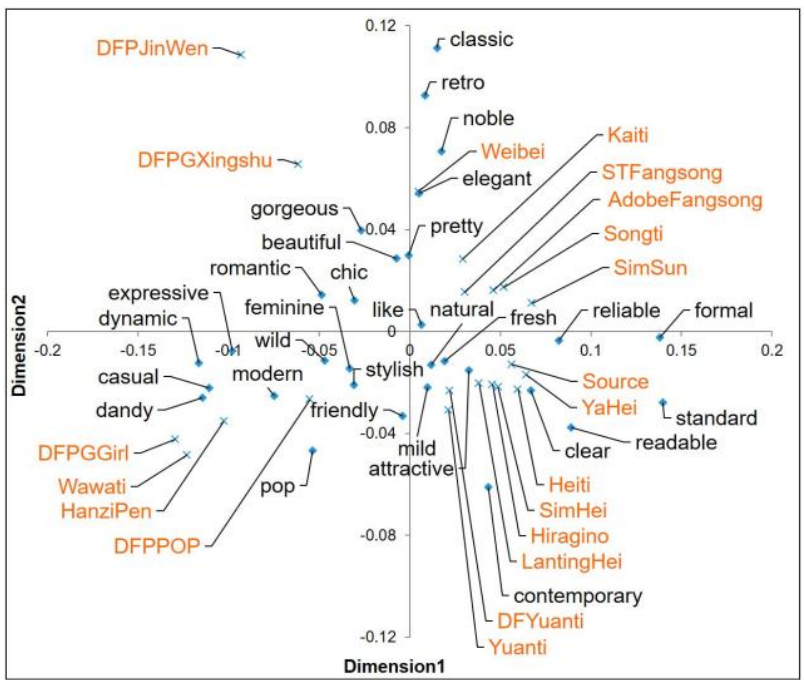

Figure 3: Emotional mapping of $20 \mathrm{TC}$ fonts 

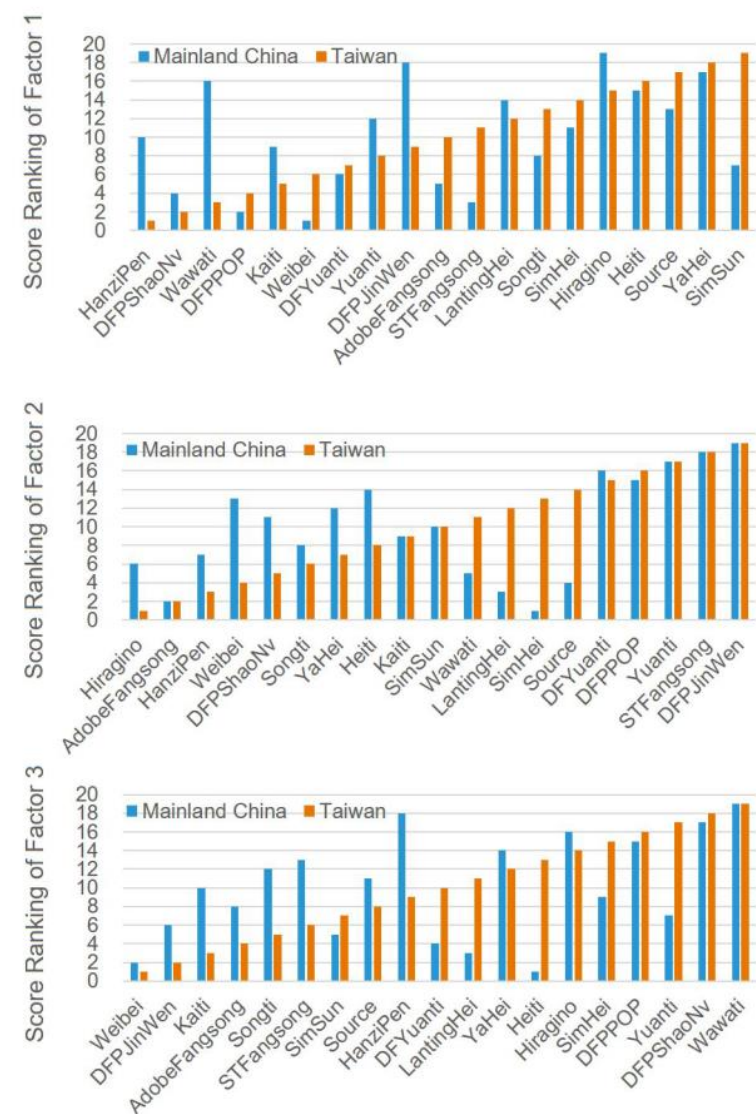

Figure 4: Factor score ranking of $19 \mathrm{SC}$ and TC fonts

contribution of the dimensions, 2 dimensions are extracted and the mapping results for SC and TC fonts are shown in Figure 2 and 3. In these two figures, most emotions have similar distribution, while the distribution of serif types and san-serif types of SC and TC fonts have opposite distributions. The people in mainland China thought Heiti types are more classic, and Songti, Fangsong, Kaiti are more contemporary. While the people in Taiwan thought Heiti types are more contemporary, and Songti, Fangsong, Kaiti are more classic.

For details, the ranking of factor scores for 19 fonts for the three factors in previous section are shown in Figure 4. For factor informality, the people in mainland China and Taiwan have different impressions about HanziPen and Wawati that have cute handwriting style. The people in Taiwan have the impressions of casual and dynamic for these two fonts, while the people in mainland China do not. Moreover, the people in mainland China think that STFangsong and SimSun are a little casual and dynamic, while the people in Taiwan do not think so. For factor practicality, mostly the people in mainland China and Taiwan have similar feelings of readable and standard for fonts. However, some Heiti and Yuanti as Source Hans, SimHei, and DFYuanti are found with affective difference between the mainland China and Taiwan. For factor times, the people in Taiwan have strong impressions of classic and retro for Kaiti, Fangsong, and Songti, while the people in mainland China think Heiti is more classic.

\section{CONCLUSION}

This research is a beginning of exploring the affective differences of typeface across cultures. The key contribution of our work is to find out that there are significant differences of typographic feelings between the people in mainland China and Taiwan. For a same design objective, it is suggested to use different types to meet the affective needs of people from different culture and life environment. The affective knowledge of Chinese typefaces is used as a guideline for Chinese font selection, and it is also utilized in automatic design system.

Considering the internationalization and multilingual needs, we will compare the affective difference among other writing systems where Chinese characters are remained as a key component, such as Kanji in Japanese. Furthermore, we will explore and verify the historical reasons among different cultures for typographic feelings in our future work.

\section{ACKNOWLEDGMENTS}

This work was supported by Grant-in-Aid for KANSEI Research of Japan.

\section{REFFERENCES}

[1] A.D. Shaikh; Psychology of Onscreen Type: Investigations Regarding Typeface Personality, Appropriateness, and Impact on Document Perception, 2007.

[2] Q.R. Qiu and K. Omura; Developing a Document Creating System for Affective Design: A Case Study in Card Design, International Journal of Affective Engineering, 15(2), pp.91-99, 2016.

[3] Q.R. Qiu, S. Watanabe and K. Omura; Images and Anatomy of Latin Typefaces, International Journal of Affective Engineering, 16(2), pp.121-130, 2017.

[4] S. Mukai; Consideration and Classification of the Factor Structure of Impression of Japanese Fonts; The 60th Annual Conference of JSSD, 2013.

[5] N. Yang and H. Ihara; Classifying the Typeface Design of Chinese Typefaces for Body Text: Sòngtĩ and hēitǐ; The Annual Conference of JSSD, 2013.

[6] J. Dobres, N. Chahine, B. Reimer, D. Gould and N. Zhao; The Effects of Chinese Typeface Design, Stroke Weight, and Contrast Polarity on Glance Based Legibility, Displays, Vol.41, pp.42-49, 2016. 